\title{
Bone Levels - From Hypothesis To Facts
}

\author{
Savitha B ${ }^{1}$, Priyanka Vhanmane ${ }^{2}$, Hiroj Bagde ${ }^{3}$, Alka Waghmare ${ }^{4}$ \\ ${ }^{I}$ (Associate Professor, Department of Periodontics, ACPM Dental College, India) \\ ${ }^{2}$ (PG Student, Department of Periodontics, ACPM Dental College, India) \\ ${ }^{3}$ (PG Student, Department of Periodontics, ACPM Dental College, India) \\ ${ }^{4}$ (Professor, Department of Periodontics, ACPM Dental College, India)
}

\begin{abstract}
The most frequent operations used for the regeneration of periodontal tissues are the flap operation, bone graft, guided tissue regeneration, and a combination of bone graft and guided tissue regeneration. In assessing the regenerated periodontal tissue, it is difficult to judge whether true regeneration has taken place without histological evaluation. The present study evaluated the clinical reliability and accuracy of bone probing measurements by transgingival probing and radiographic bone level by RVG in assessment of bone level by comparing those results with surgically confirmed bone levels. The study concluded that SBL provides actual bone level assessment but it requires surgical re-entry which might hamper wound healing and may also be inconvenient for the patients. Hence bone level measurement by transgingival probing which highly corelates with bone levels measured surgically, is most reliable method for assessing bone levels before and after any surgical procedures.
\end{abstract}

Keywords - bone level, intrabony defect, radio visuo graph, stent, trans gingival probing.

\section{Introduction}

The ultimate goal of periodontal therapy is not only to stop the periodontal disease, but also to regenerate the destroyed periodontal tissue and enable it to function normally. The regeneration of periodontal tissue involves the formation of new cementum, the functional insertion of periodontal ligament fiber into it, and the subsequent increase in the bone level. The most frequent operations used for the regeneration of periodontal tissues are the flap operation, bone graft, guided tissue regeneration, and a combination of bone graft and guided tissue regeneration.

In assessing the regenerated periodontal tissue, it is difficult to judge whether true regeneration has taken place without histological evaluation. A number of parameters have been used for the evaluation of the regenerated bone level ${ }^{1,2}$. The most accurate method of assessing the bone level, of course, is to elevate the flap and measure the bone level directly. However, this method causes discomfort to the patient and can damage the regenerated tissues. Therefore, many studies have been conducted to find an alternative method that can be used to assess the bone level clinically with accuracy and reliability ${ }^{2-7}$. For example, among the methods that have been proposed so far are the probing depth measurement, various radio-graphic bone level measurements, and the bone probing technique. In subsequent studies, it has been shown that the bone probing measurement is closely correlated with the actual bone level.

Marshall-Day and Shourie and Ramfjord demonstrated the clinical usefulness of radiographic assessment of bone level ${ }^{8,9}$ while Suomi et al and Burnett suggested the limitation of this method because of its tendency for underestimating actual bone $\operatorname{loss}^{6,10}$. Renvert et al reported that the attachment level, bone probing level, and surgically confirmed bone level had a high correlation in the assessment of intrabony defects treated with regenerative therapy, while the radiographic bone level did not ${ }^{2}$. However, use of RVG might provide reliable accuracy.

The present study evaluated the clinical reliability and accuracy of bone probing measurements by transgingival probing and radiographic bone level by RVG in assessment of bone level by comparing those results with surgically confirmed bone levels.

\section{Subjects and sites}

\section{MATERIALS AND METHODS}

Patients aged 20-45 years with chronic generalized periodontitis who were scheduled for periodontal flap surgery from the OPD of department of Periodontics, ACPM Dental College and hospital were selected for the study. After obtaining clearance from ethical committee and a written consent from the patients and they received oral hygiene instructions, scaling and root planing prior to study. 56 sites were studied and the mesial and distal surface was probed in the teeth planed for surgery. 


\section{PROCEDURES}

Study models of the areas to be studied were made from alginate impressions, and onlay type stents were fabricated using auto-curing resin for each tooth. A stainless steel wire was incorporated in the stent for radio-opacity. Vertical locating grooves were made in the buccal aspect with burs for the proper guidance and orientation of the periodontal probe.

For the measurement of bone probing depth by transgingival probing a Williams graduated probe was used. Local anesthesia was administered prior to measuring bone probing depth. Stent was positioned in place and the attachment level was recorded as the distance from the wire, which was taken as the reference line till the base of the pocket. The deepest depth at which the probe met strong resistance from contact to the bone was recorded and perpendicular to it probing was done.(Fig 1a and Fig 1b) Bleeding points so obtained gave the levels of bone (TGP) ${ }^{11}$.

For the radiographic evaluation (RBL) RVG (Radio visuo graph) was used while the stent was in place. The distance from the stent to bone was measured.(Fig 2)

For actual measurement of bone levels at surgery (SBL), the flap was elevated and defects debrided. The stent was placed and the probe was oriented. The distance from the reference line of the stent to the deepest part of the defect was measured was evaluated. (Fig 3)

\section{Analysis of mean data}

\section{RESULTS}

Mean radiographic bone level, overall surgical bone level and mean transgingival bone probing levels are depicted in Table 1.

The graphical representation of the values is depicted in Graph 1.

The overall coefficient of correlation of SBL with TGP was 0.97 and with RBL was 0.96

\section{FIGURES AND TABLES}

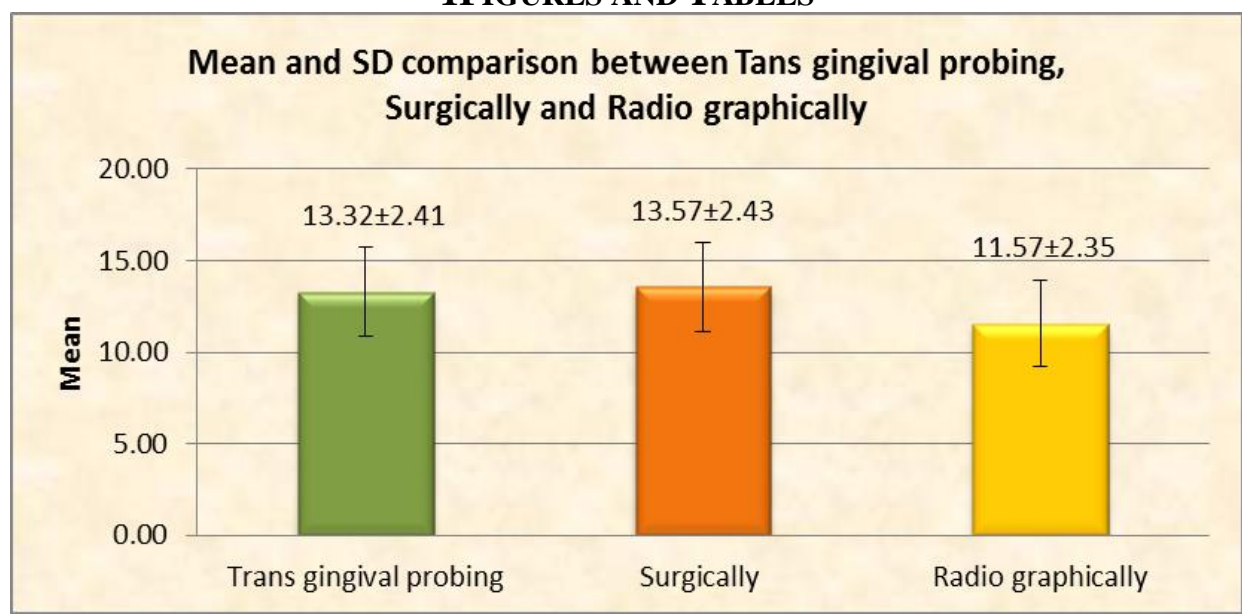

Graph 1: Mean and SD comparison between trans gingival probing, surgically and radio graphically

\begin{tabular}{|c|c|c|c|}
\hline VARIABLE & $\mathrm{MEAN} \pm \mathrm{SD}$ & Mean difference & $\mathrm{P}$ value \\
\hline Trans gingival probing & $13.32 \pm 2.41$ & \multirow{2}{*}{$0.25 \pm 0.02$} & \multirow{2}{*}{0.846} \\
\hline Surgically & $13.57 \pm 2.43$ & & \\
\hline Trans gingival probing & $13.32 \pm 2.41$ & \multirow[b]{2}{*}{$1.75 \pm 0.06$} & \multirow[b]{2}{*}{$<0.0001 *$} \\
\hline Radio graphically & $11.57 \pm 2.35$ & & \\
\hline Surgically & $13.57 \pm 2.43$ & \multirow{2}{*}{$2.00 \pm 0.08$} & \multirow{2}{*}{$<0.0001^{*}$} \\
\hline Radio graphically & $11.57 \pm 2.35$ & & \\
\hline
\end{tabular}

$\mathrm{p}$ value $<0.01$ - statistically significant

Table 1: Mean and SD comparison between trans gingival probing, surgically and radio graphically 


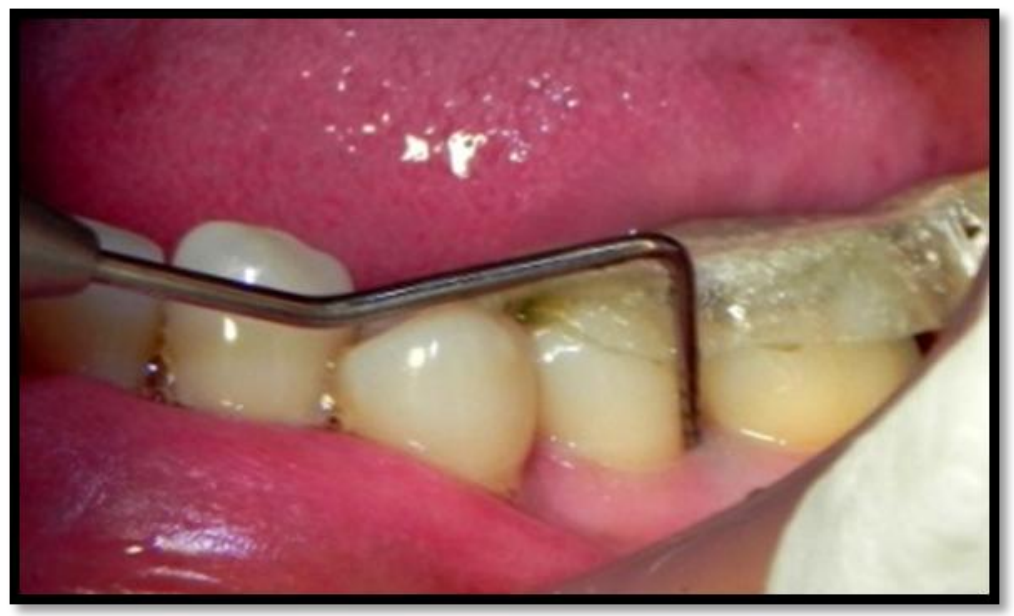

Figure 1a - Bone level assessment by Trans Gingival Bone Probing (TGP)- Probe is penetrated vertically until resistance is felt.

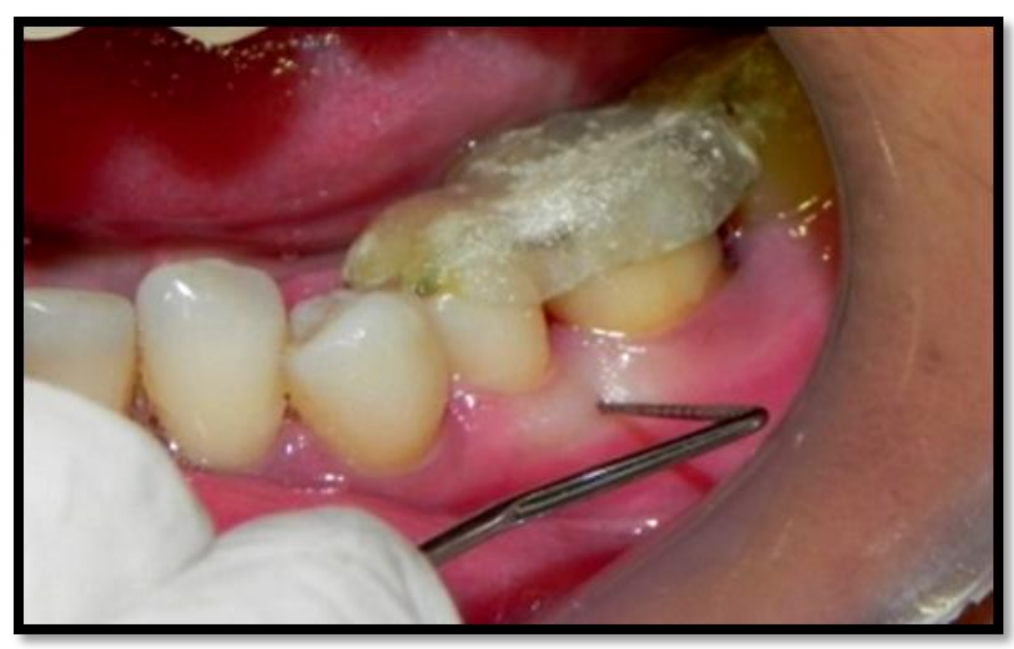

Figure 1b- Bone level assessment by Trans Gingival Bone Probing (TGP)- Probe is penetrated horizontally and bleeding point marked.

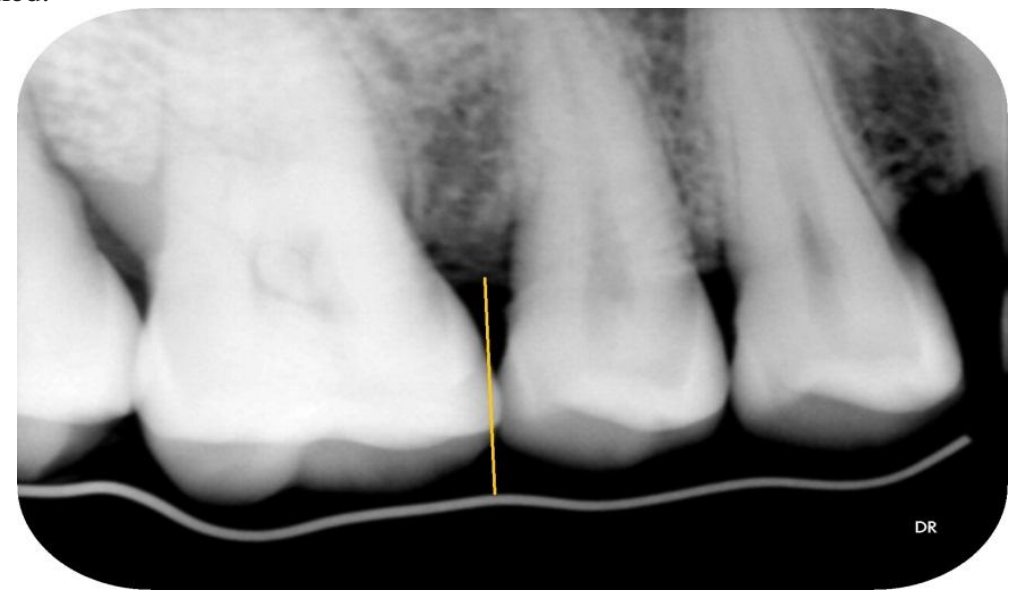

Figure 2 - Bone Level assessment by Radio Visuo Graph (RBL) 


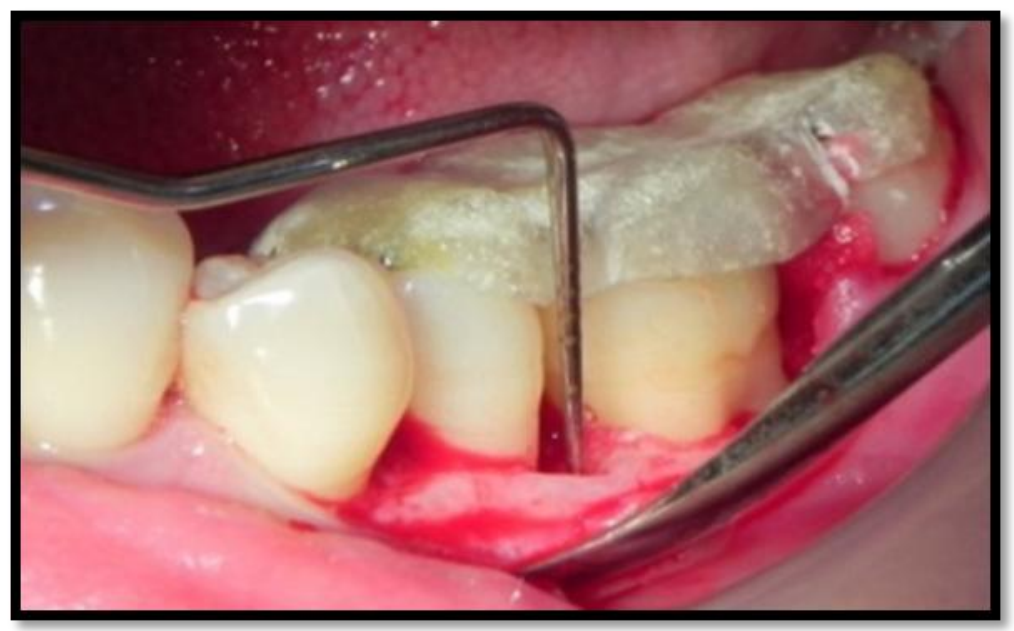

Figure 3 - Bone level assessment by Surgical Flap Elevation (SBL)

\section{DISCUSSION}

The aim of the present study was to compare the bone levels measured by transgingival probing (TGP) and radiographically by RVG (RBL) with the bone level measured surgically (SBL).

For consistent measurement, custom-made stents were used. Hassell et al (1972) reported that the accuracy of probing in evaluation of attachment level might suffer if the site and direction of the probing were not consistent ${ }^{12}$. Same problem may occur in the evaluation of regenerative therapy. Therefore, a consistent reference and orientation for probing are needed. An onlay-type stent which can guide the probe to an exact location with a proper orientation may be used instead. Clark 1987 and Badersten et al 1984 reported that the onlay-type stent could enhance the reliability of attachment level assessment ${ }^{13}$.

Probing depth is defined as the distance from gingival margin to base of the periodontal pocket. There are various histological studies which studied the depth of the probe insertion as compared to normal surgical measurement. They suggested that bone level is not reached by the normal probing and hence trans-gingival probing was opted for the same.

Bone probing measurement requires the insertion of a probe until the tip contacts the bone, a technique that Easley termed the bone sounding technique. In this method, a probe is penetrated horizontally and vertically through the anesthetized gingiva down to the bone in order to assess the bone morphology11. Greenberg et al. referred to this technique as transgingival probing and reported that the vertically probed bone level and the surgically confirmed bone level were closely correlated ${ }^{3}$.

In the present study, on comparison between TGP and RBL there was statistically significant difference with a high correlation (0.96), which is in accordance with Renvert et al 1981 who reported that radiographic bone height showed lower degrees of correlation with bone probing depth.

The difference between TGP and SBL was not statistically significant. These results are in agreement with Renvert et al in 1981 who reported that a high degree of correlation was found between bone probing depth and surgical entry levels with correlation of 0.752. Similar study by Ursell et al in 1989 reported that transgingival probing was an accurate method of measuring alveolar bone levels ${ }^{7}$.

On comparison between RBL and SBL a statistically significant difference was obtained which is in accordance with Shrout et al (1996) ${ }^{14}$ who reported radiographic determination of bone loss lacks accuracy which may be attributed to patterns of bone destruction which vary with the severity of the disease, especially in interproximal areas. Burnett 1971 reported the tendency of radiographs to underestimate actual bone loss ${ }^{10}$.

The results of this study suggest that, a RBL underestimates the bone level measurements compared to TGP and SBL which is in accordance with Hyun-Young Kim et al (2000) who concluded that there was minimal difference between the bone probing measurement and actual surgical bone level ${ }^{15}$.

With radiograph there are chances of radiation exposure also, hence it might not be a reliable method for assessing bone levels.

SBL provides actual bone level assessment but it requires surgical re-entry which might hamper wound healing and may also be inconvenient for the patients.

Hence bone level measurement by transgingival probing which highly co-relates with bone levels measured surgically, is most reliable method for assessing bone levels before and after any surgical procedures. 


\section{REFERENCES}

[1]. Kim HY, Yi SW, Choi SH, Kim CK. Bone probing measurement as a reliable evaluation of the bone level in periodontal defects. J Periodontol2000;71:729-735.

[2]. Renvert S, Badersten A, Nilveus R, Egelberg J. Healing after treatment of periodontal intraosseous defects. I. Comparative study of clinical methods. J Clin Periodontol 1981;8:387-399.

[3]. Greenberg J, Laster L, Listgarten MA. Transgingival probing as a potential estimator of alveolar bone level. J Periodontol 1976;47:514-517.

[4]. Isidor F. Clinical probing and radio-graphic assessment in relation to the histologic bone level at oral implants in monkeys. Clin Oral Implants Res 1997;8:255-264.

[5]. Mealey BL, Neubauer MF, Butzin CA,Waldrop TC. Use of furcal bone sounding to improve accuracy of furcation diagno-sis.J Periodontol 1994;65:649-657.

[6]. Suomi JD, Plumbo J, Barbano JP. A comparative study of radiographs and pocket measurements in periodontal dis-ease evaluation. J Periodontol 1968;39:311-315.

[7]. Ursell MJ. Relationships between alveolar bone levels measured at surgery, estimated by transgingival probing and clinical attachment level measurements. J Clin Periodontol1989;16:81-86.

[8]. Marshall-Day CD, Shourie KL. A roentogenographic survey of periodontal disease in India. J Am Dent Assoc 1949;39:572-588.

[9]. Ramfjord SP. Indices for prevalence and incidence of periodontal disease. J Periodontol 1959;30:51-59.

[10]. Burnett E W. Limitation of the roentgenogram in periodontal diagnosis. J Periodontol 1971;42:293-296.

[11]. Easley JR. Methods of determining alveolar osseous form. J Periodontol 1967;38:112-118.

[12]. Hassell TM, Germann MA. Periodontal probing: Inter-investigator discrepancies and correlations between probing force and recorded depth. Helv Odontol Acta 1972; 17:38-42.

[13]. Badersten A, Nilveus R, Egelberg J. Reproducibility of probing attachment level measurements. J Clin Periodontol 1984;11:475485 .

[14]. Shourt MK, Powell BJ, Hildebolt CF, Vannier MW, Ahmed NM. Digital radiographic image-based bone level measurements: Effect of film density. J Clin Periodontol 1993;20:595-600.

[15]. Hyun-young Kim, et al. Bone probing measurement as a reliable evaluation of the bone level in periodontal defects. J Periodontol 2000; 5: 729-735 